\title{
THE FCF FLUIDS INTEGRATED RACK: MICROGRAVITY FLUID PHYSICS EXPERIMENTATION ON BOARD THE ISS
}

\author{
Frank G. Gati \\ Myron E. Hill \\ National Aeronautics and Space Administration \\ Glenn Research Center \\ Cleveland, Ohio 44135
}

\begin{abstract}
$\underline{\text { Abstract }}$
The Fluids Integrated Rack (FIR) is a modular, multiuser scientific research facility that will fly in the U.S. laboratory module, Destiny, of the International Space Station (ISS). The FIR will be one of the racks that will constitute the Fluids and Combustion Facility (FCF) ${ }^{1}$. The ISS will provide the FCF and therefore the FIR with the necessary resources, such as power and cooling, so that the FIR can carryout it's primary mission of accommodating fluid physics science experiments. This paper discusses the mission, design, and the capabilities of the FIR in carrying out research on the ISS.
\end{abstract}

\section{Introduction}

The FCF is being designed to accommodate a wide range of combustion and fluids physics experiments. At completion the FCF will occupy three International Standard Payload Racks (ISPR's) as shown in Figure 1. The Combustion Integrated Rack (CIR) will be the first

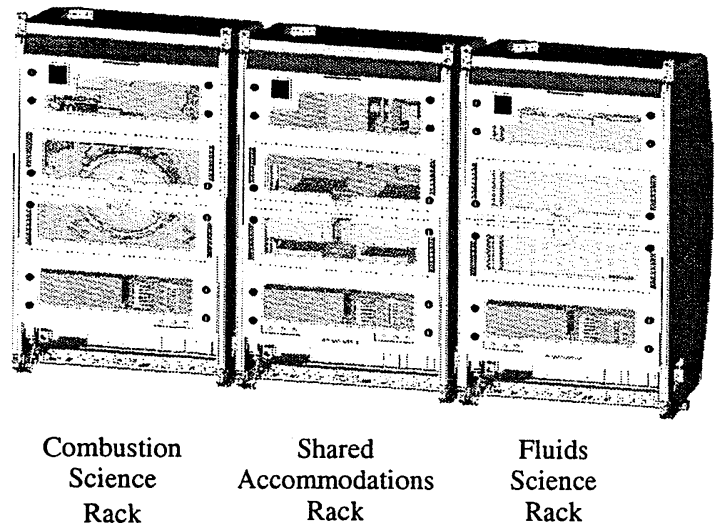

Figure 1 - Fluids and Combustion Facility

of the FCF racks onboard the ISS. The CIR will be launched on Utilization Flight (UF) 3 scheduled in

Copyright $(\subset) 2001$ by the American Institute of Aeronautics and Astronautics, Inc. No Copyright is asserted in the United States under Title 17, U.S. Code. The U.S. Government has a royalty-free license to exercise all rights under the copyright claimed herein for Governmental Purposes. All other rights are reserved by the copyright owner.
January 2005. The primary mission of the CIR is to accommodate combustion experiments involving liquid, solid and gaseous fuels ${ }^{2}$. The FIR is the next FCF rack to be launched. The FIR is scheduled for launched in September of 2005 on UF-5. The Shared Accommodations Rack (SAR) will be the last rack to be launched on UF 6 scheduled in January of 2006. The SAR has a two-fold mission. The SAR's design is similar to the FIR enabling it to accommodate experiments from various science disciplines. In addition to accommodating experiments, the SAR will provide additional resources for image acquisition, image analysis and data storage that can be utilized by experiments in the FIR and CIR. Avionics from the CIR and FIR may also be offloaded to the SAR to increase volume and mass available for experimental hardware in the FIR and CIR.

One of the key design drivers for the FIR is to provide common laboratory diagnostic hardware, in a flexible environment, in order to accommodate a variety of imaging techniques commonly used in fluid physics experiments. With this in mind the FIR is being designed to support various diagnostic techniques such as: Video Imaging, Video Microscopy, Light Scattering, Shadowgraphy, Particle Image Velocimetry, Interferometry, IR-imaging, Confocal Microscopy, Laser Tweezers, and Surface Profilometry. In addition, the design of the FIR infrastructure is such that experiment unique cameras, light sources and optical hardware can be accommodated through standard interfaces if the FIR diagnostics tools are not sufficient for a particular diagnostic technique. In order to provide a flexible environment that can accommodate the various experimental test cells and the required diagnostics, the FIR provides a large volume for experimental payloads. Within this experiment volume, experimental hardware can be precision-mounted directly to the FIR optics bench.

The FCF/FIR systems are being designed to maximize the amount of science that can be done on-orbit. Experiments will be designed and operated to efficiently progress through the test matricies. This will be through a combination of highly automated systems, on-orbit physical component change-outs or processing by the crew, and software updates via ground

This is a preprint or reprint of a paper intended for presentation at a conference. Because changes may be made before formal publication, this is made available with the understanding that it will not be cited or reproduced without the permission of the author. 
commanding or by the crew. Another key feature that is carefully coordinated will be the on-orbit storage and downlinking of image data.

The FIR is being designed to allow easy manipulation, installation and removal for FIR hardware by the ISS crew. The FIR can be operated by an ISS crew member through a laptop computer mounted outside of the rack. While the ISS crew will be available for experiment operations, their time will be limited so the FCF is being designed for both autonomous and remote control operations. Control of the FCF will be primarily through the Telescience Support Center (TSC) at the Glenn Research Center.

\section{Mission: Fluids Physics Research}

The Fluids and Combustion Facility will be a permanent facility onboard the ISS. In support of NASA's Human Exploration and Development of Space (HEDS) Microgravity Research Program objectives, the FCF will accommodate and facilitate sustained, systematic Microgravity Fluid Physics and Microgravity Combustion Science experimentation on the ISS.

The FCF is being designed for a ten-year life with the ability to extend that life to fifteen years through hardware maintenance and hardware replacement. The goal of FCF is to be able to accommodate a minimum of ten experiments per year in each of the areas of Fluids Physics and Combustion science.

The primary mission of the FIR is to accommodate experiments from five major.fluids physics disciplines: Complex Fluids; Multiphase Flow and Heat Transfer; Interfacial and Capillary Phenomena; Colloids, and Dynamics and Stability. Furthermore, FIR's flexibility (i.e. large volume for experimental hardware, easily reconfigurable diagnostics, customizable software) allows accommodation of experiments from other disciplines such as biotechnology.

\section{Carrier - ISS}

The Fluids and Combustion Facility will be located in the U.S. laboratory module, Destiny, of the International Space Station (ISS). Destiny is an environmentally controlled, pressurized laboratory. Within Destiny there are 24 International Standard Payload Rack (ISPR) locations, 13 of these are allocated for research. The FCF will occupy three contiguous research locations. The ISS supplies the necessary resources to conduct experiments at these rack locations. Resources include power, cooling water, gases, vacuum and command and data handling. The estimated steady state resource allocation to FIR by ISS is shown in Table 1. Steady state operations commence once ISS assembly is complete. The actual allocation of resources provided to FIR during any time interval, however, may differ. Resource allocation will be based on all the activities being conducting on the ISS in the same time frame.

\begin{tabular}{|l|c|}
\hline \multicolumn{2}{|l|}{$\begin{array}{l}\text { Table 1 Estimate Steady State ISS Resources } \\
\text { Allocated to FIR }\end{array}$} \\
\hline $\begin{array}{l}\text { On-Orbit Volume (rack } \\
\text { equivalent volume) }\end{array}$ & $\begin{array}{c}\text { 1.0 Facility ISPR plus 1.0 } \\
\text { m3/(35 ft3) stowage vol. }\end{array}$ \\
\hline $\begin{array}{l}\text { Up Mass (kilograms } \\
\text { per year)/(pounds per } \\
\text { year) }\end{array}$ & $\begin{array}{c}750 \mathrm{kgs} / \\
(1653 \mathrm{lbs})\end{array}$ \\
\hline $\begin{array}{l}\text { Down Mass (kilograms } \\
\text { per year)/(pounds per } \\
\text { year) }\end{array}$ & $\begin{array}{l}750 \mathrm{kgs} / \\
(1653 \mathrm{lbs})\end{array}$ \\
\hline $\begin{array}{l}\text { Up Volume (cubic } \\
\text { meters per year)/(cubic } \\
\text { feet per year) }\end{array}$ & $1.6 \mathrm{~m} 3 /(56 \mathrm{ft} 3)$ \\
\hline $\begin{array}{l}\text { Down Volume (cubic } \\
\text { meters per year)/(cubic } \\
\text { feet per year) }\end{array}$ & $1.6 \mathrm{~m} 3 /(56 \mathrm{ft} 3)$ \\
\hline $\begin{array}{l}\text { Energy (kilowatt hours } \\
\text { per year) }\end{array}$ & 3200 \\
\hline $\begin{array}{l}\text { Crew Time (hours per } \\
\text { year) }\end{array}$ & 180 \\
\hline $\begin{array}{l}\text { Communications } \\
\text { Downlink (Terabits per } \\
\text { year) }\end{array}$ & 41.1 \\
\hline $\begin{array}{l}\text { Communications } \\
\text { Uplink (Terabits per } \\
\text { year) }\end{array}$ & $1.72 \times 10(-4)$ \\
\hline
\end{tabular}

\section{$\underline{\text { FIR }}$}

The FIR will utilize six major subsystems to accommodate the broad scope of fluids physics experiments. The major subsystems are: structural, environmental, electrical, gaseous, command and data management and diagnostics. These subsystems combined with payload unique hardware will allow the FIR to conduct world-class science.

\section{Structural Subsystem}

The foundation of the FIR's structural subsystem is the ISPR. The ISPR will contain all of the FIR hardware and provide a standard interface to Destiny. Enclosing the rack, in the front, will be two bi-fold doors. The doors open from the middle up and the middle down. The rack, along with the doors, provides acoustic emission attenuation, minimizes air/thermal exchange with the cabin, reduce airborne contaminate migration into the rack and prevents stray light from entering or leaving the rack. 
The Active Rack Isolation System (ARIS) enhances the micro-gravity environment for experiments in the FIR. ARIS, mounted in the ISPR, minimizes vibratory transmission to and from the rack. ARIS utilizes three accelerometers to measure the micro-gravity environment in the rack. Based on the measured accelerations ARIS applies reactive forces, between the FIR and ISS, through eight actuators to minimize transmission of vibratory disturbances.

The centerpiece of the FIR structural subsystem is the optics bench. The optics bench provides a mounting surface for FIR light sources and avionics packages on the back of the bench and for payload hardware on the front of the bench. Depending on the use of the FIR diagnostics, the optics bench will be able to accommodate up to $125 \mathrm{~kg}$ of payload hardware. The front of the optics bench provides T-Slots and 1/4-28 threaded holes, as shown in Figure 2, for mounting of

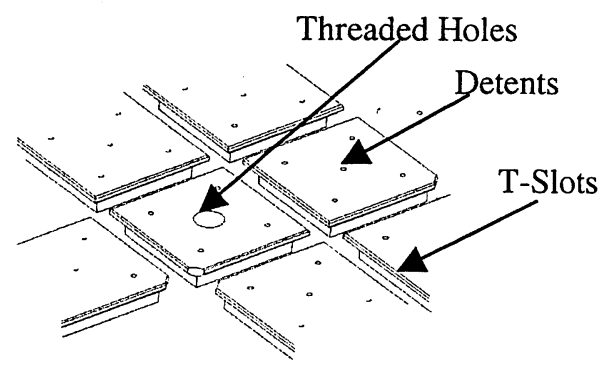

Figure 2 - Optics Bench Front Surface

payload hardware. The T-slots enable precise mounting of payload hardware; including optical components. The T-Slots are spaced on $50 \mathrm{~mm}$ centers. Detents on $12.5 \mathrm{~mm}$ centers will enable precise indexing when positioning hardware using the T-slots. The FIR designed quick-connect mounting latches, shown in Figure 3, can be used for mounting payload hardware to position and attach hardware to the optics bench without using tools.

The optics bench is designed so it can be translated out

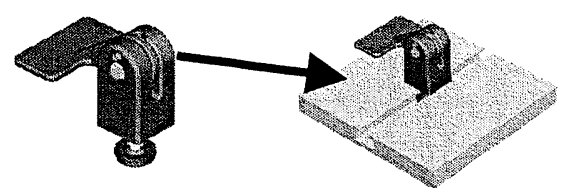

Figure 3 - Quick-connect Mounting Latch

of the rack and rotated forward, as shown in Figure 4, to allow the crew easy access to hardware mounted on the optics bench. During powered operations the FIR optics bench is in the stowed position and the rack doors are closed. In the un-powered mode, the rack doors can be opened and the optics bench translated forward. Fully translated out, the front surface of the bench will extend past the front of the rack slightly into the aisle way of the cabin providing easy access to the front of the bench. The bench can be rotated forwarded ninety degrees providing access to the back of the bench for procedures such as replacing FIR hardware on the back of the bench or routing a fiber optic cable from the light sources on the back of the bench to payload hardware on the front of the bench.

\section{Environmental Subsystem}

The environmental subsystem will utilize air and water to remove heat generated by the FIR and payload hardware. Air will be circulated throughout the rack and will be cooled by the Air Thermal Control Unit (ATCU), an air-water heat exchanger located in the top of the rack. The design point for the air-cooling system is $1650 \mathrm{~W}$ at 220 cubic feet per minute, however, the system can operate at other heat loads and air flow

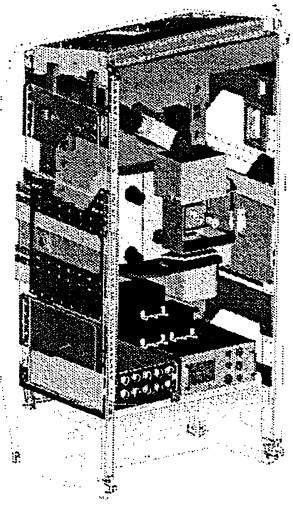

Stowed

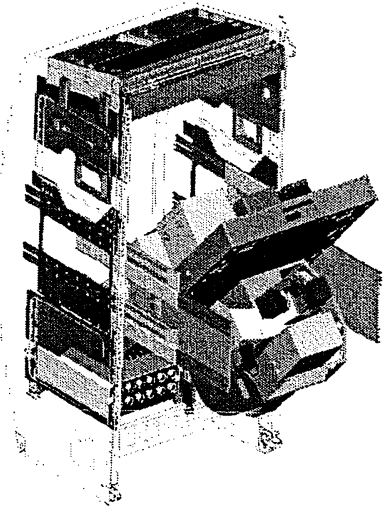

Fully Translated and Partially

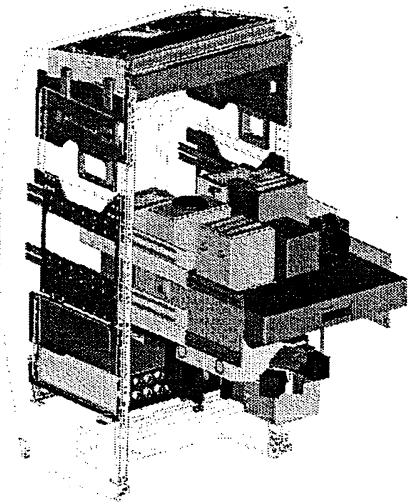

Fully Translated and Fully Rotated

Figure 4 - FIR Optics Bench Deployment

utilizing the T-slots. These latches will allow the crew 
rates. Cool air, nominally at $22.5{ }^{\circ} \mathrm{C}$, will leave the heat exchanger and flows over the front of the optics bench cooling payload hardware as well as the FIR diagnostics located on the front of the bench. Heat rejection to the air on the front of the bench is nominally limited to 500 W. After cooling the hardware on the front of the bench, the air will cool the hardware located below and on the back of the optics bench. The warm air then returns to the ATCU through the optics bench (Figure 5).

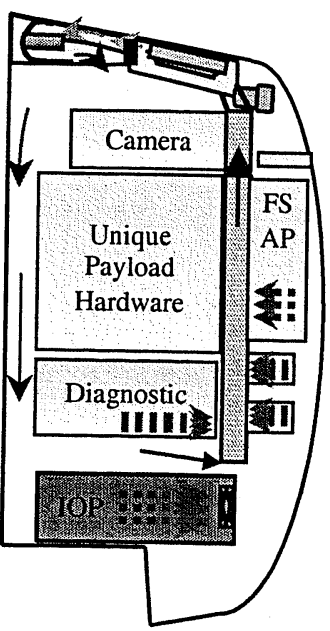

Figure 5 - FIR ATCS
Cooling water from the ISS moderate temperature loop enters the rack and is split into two cooling loops. One loop is dedicated to the FIR hardware the other loop is for payload hardware. Each loop is capable of removing $3 \mathrm{~kW}$ of heat. The water inlet temperature to the rack is nominally $16.6^{\circ} \mathrm{C}$. The ISS regulates the water mass flow rate through the rack in order to maintain nominally a $20{ }^{\circ} \mathrm{C}$ delta between the water inlet and outlet temperatures. Payloads have access to the cooling water through the Water Interface Panel located on the right side of the rack.

\section{Gas Interface Subsystem}

The FIR will provide payloads with access to the ISS gaseous nitrogen and vacuum systems through the Gas Interface Panel (GIP) located on the left side of the rack. These systems are available to support experiment operations such as the purging of experimental test cells and pressurizing or creating flows within experimental test cells.

The ISS does not regulate the gaseous nitrogen system supply pressure and temperature. The supply pressure and temperature can vary from 517 to $827 \mathrm{kPa}$ and from $15.5{ }^{\circ} \mathrm{C}$ to $45{ }^{\circ} \mathrm{C}$ respectively. The FIR provides a shutoff valve isolating payload hardware from the ISS gaseous nitrogen system. The payload must provide flow control and pressure regulation as required. The maximum mass flow rate is $5.43 \mathrm{~kg} / \mathrm{hr}$.

FIR provides payloads access to the two ISS vacuum systems, the Vacuum Exhaust System (VES) and the Vacuum Resource System (VRS). The VES has a higher throughput then the VRS and is intended for pulling a chamber or system from pressures up to 276 $\mathrm{kPa}$ down to a vacuum $(0.13 \mathrm{~Pa})$. The VRS can be used to hold a chamber or system at a vacuum $(0.13 \mathrm{~Pa})$.
Payloads will be able to access the ISS vacuum systems through the FIR provided quick disconnects located in the GIP.

\section{Electrical Subsystem}

The Electrical Power Control Unit (EPCU) is the heart of the electrical subsystem. All power from ISS will flow through the EPCU. The EPCU will provide power management and control functions, as well as fault protection. The EPCU will take 120 VDC from ISS power bus to provide six 120 VDC $4 \mathrm{~A}$ arıd forty-eight 28 VDC 4 A fault protected circuits to the FIR and payload hardware. Payload hardware will have access to three of the $120 \mathrm{VDC}$ circuits through connectors located near the left front rack post and six of the 28 VDC circuits at the PI power/data connectors, shown in Figure 6, on the optics bench.

\section{Command and Data Management Subsystem}

The FIR Command and Data Management subsystem (CDMS) provides command and data handling for both facility and payload hardware. The main components of the FIR CDMS are the Input Output Processor (IOP), the Image Processing and Storage Unit (IPSU), the Fluids Science Avionics Package (FSAP) and the PI FSAP.

The Input Output Processor (IOP) will provide the link

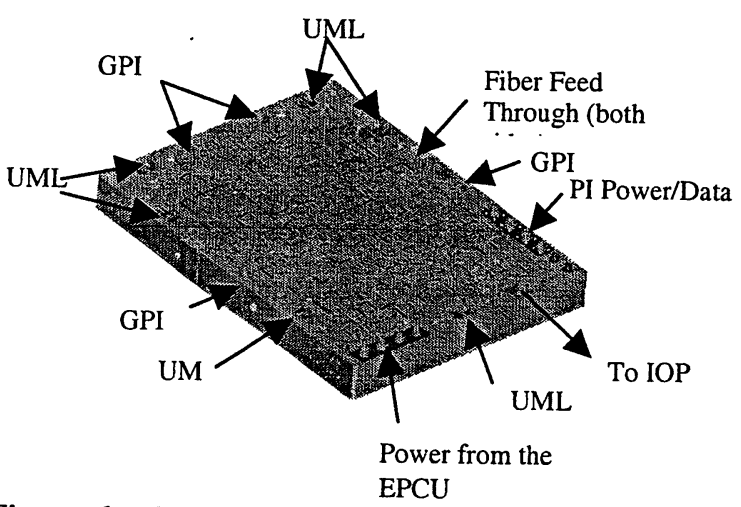

Figure 6 - Optics Bench Front Electrical, Command and Data Handling Interfaces

from the FIR to the ISS command and data management system via the MIL-STD-1553B, Ethernet, analog video and the High Rate Data Link interfaces. The MIL-STD-1553B is used for command and control as well as health and status communications between FIR and ISS. The other interfaces support data transfer from the FIR to ISS for downlinking to the ground. The Input Output Processor provides the overall command and data management functions for the FIR. The IOP controls the core facility subsystems such as the ARIS, EPCU and the environmental control subsystem. The IOP will receive and store science data from the FSAP, image data from the IPSU's and ancillary rack data. The IOP will use the ancillary rack 
data to monitor the health and status of the rack hardware. The IOP will store data on two removable 182 GB hard drives. This data can be downlinked or the hard drives can be removed and replaced with new ones. The IOP can route analog video directly to the ISS for downlinking or storage on an ISS video recorder.

The FIR will be able to accommodate two Image Processing and Storage Units (IPSU). The Image Processing and Storage Units will perform diagnostic control and image processing and storage functions. The Fluids Science Avionics Package controls each IPSU. An IPSU will be able to provide control for a digital camera, receive image data from the camera and process or compress image data as necessary. Payloads can use existing FIR software or generate custom software to process and compress image data. The IPSU can receive images from a digital camera through a custom Serial Data Link (SDL) interface or through a standard IEEE-1394 interface. The serial data link converts image data transmitted from a digital camera through fiber back to a digital signal so it can be processed by the IPSU. Cameras with an IEEE-1394 interface can be controlled through this interface. Cameras utilizing the SDL data interface can be controlled utilizing the Control Area Network (CAN) bus provided through either the Universal Mounting Location (UML) or the Generic Package Interface (GPI) connectors (Figure 6). The IPSU will be capable of receiving raw image data at $64 \mathrm{MB} / \mathrm{s}$. At this rate the IPSU will be capable of receiving a 1024 pixel by 1024 pixel, 16 bit image at 30 frames per second. The IPSU will be capable of performing automated realtime image analysis in order to support real-time activities such as object tracking. Payloads can utilize existing FIR software or generate custom software for object tracking. The IPSU has two $18 \mathrm{~GB}$ hard drives for image storage. Once the data is stored the IPSU will be capable of post processing images.

The Fluids Science and Avionics Package (FSAP) will provide the primary control in carrying out an experiment. Through the FSAP the payload will be able to execute an experiment by controlling the FIR diagnostics and avionics packages as well as payload hardware. Payload developers will be able to download their own custom software into the FSAP for experiment control. The FSAP will be able to command and control the IPSU's via Ethernet. The FSAP provides a standard set of computer data acquisition and control functions for use by the payload. The FASP will provide the following: four channels of motion control, 32 analog to digital channels, 8 digital to analog channels, 24 channels each of digital input and output, external and internal triggers, 2 channels of RS-422, analog frame grabber and CAN bus. The FSAP has two $18 \mathrm{~GB}$ hard drives for storing analog and digital data as well as analog video images captured by the analog frame grabber. Payload hardware on the front of the optics bench will have access to the FSAP through the PI power/data connectors located on the front of the optics bench (Figure 6).

The PI-FSAP is a supplemental unit for the FSAP. Due to the great variation in requirements for fluids physics experiments it is anticipated that some payloads will have unique command and or data requirements that the FSAP cannot accommodate. The PI-FSAP is being designed to accommodate these additional requirements. The PI-FSAP consists of a microprocessor, Ethernet and CAN bus interfaces and three empty card slots. The PI-FSAP can be populated with payload unique boards on the ground and launched with the payload. When the payload is installed the PIFSAP is installed on the back of the optics bench. A custom cable from the PI-FSAP would be routed through the cable feed-through in the optics bench to payload hardware located on the front of the bench.

\section{Diagnostics Subsystem}

With the initial deployment of the FIR and through facility upgrades, the FIR will provide a suite of cameras and illumination sources to support a wide range of diagnostic capabilities typically required by fluids physics experiments. The FIR cameras will offer color and black and white imaging. These cameras will be capable of frame rates up to 32,000 frames per second. Lenses for these cameras will provide for macroscopic imaging. The FIR will provide two illumination sources, $532 \mathrm{~nm} \mathrm{Nd:YAG} \mathrm{laser} \mathrm{and} \mathrm{a}$ white light source for use with the FIR or payload cameras.

The FIR color camera is a 24 bit, 3-chip Charged Coupled Device (CCD) analog color camera. The camera has a one-third inch array with a pixel density of 768 pixels $\times 484$ pixels. The camera's frame rate is settable up to 30 frames per second (fps) and shutter speed is settable from $1 / 60$ of a second down to $1 / 10,000$ of a second. In auto shutter mode, shutter speeds down to $1 / 50,000^{\text {th }}$ of a second can be achieved. The color camera will be controlled by the FSAP over the CAN bus. Images from the color camera will be sent to the FSAP via a RS-170A signal for storage. The color camera has a small remote head, $32 \mathrm{~mm}$ (W) x 40 $\mathrm{mm}(\mathrm{H}) \times 40 \mathrm{~mm}(\mathrm{D})$, to facilitate camera integration in congested areas. The color camera utilizes a standard C-mount for lenses. Due to the size of the camera it will not have any mechanical attachment points other then to the lens therefore the camera can be physically mounted or secured through the lens.

The FIR high resolution digital black and white camera package is capable of, at a minimum, imaging $30 \mathrm{fps}$ of 
a 1024 pixel $x 1024$ pixel image with a dynamic range of 12 bits. Through binning, frame rates up to $110 \mathrm{fps}$ can be achieved. The high resolution camera has settable frame rates and shutter speeds. The high resolution camera will be controlled by the IPSU through the CAN bus. The high resolution camera package converts the digital images so they can be sent to the IPSU, via fiber, for storage and processing as required. The camera will utilize a four tab quick disconnect mount that will allow easy change out of a lens. The camera may be mounted to the FIR optics bench or integrated into payload hardware.

To meet higher frame rate requirements, the FIR will be providing a digital black and white Ultra High Frame Rate (UHFR) camera capable of recording $1000 \mathrm{fps}$ at a minimum pixel density of 512 pixels x 512 pixels, 8 bits/pixel; and 32,000 fps at a minimum pixel density of 128 pixels by 32 pixels, 8 bits/pixel. Electronic shuttering will allow exposure times, independent of the frame rate, down to 10 microseconds. While images are being recorded they will be stored in memory onboard the camera. Once the camera memory is full the images will be transferred to the IPSU via an IEEE1394 interface. The camera will be controlled by the IPSU via the IEEE-1394 interface. The camera will utilize a 4 tab quick disconnect mount that will allow easy change out of a lens. The camera may be mounted to the FIR optics bench or integrated into payload hardware.

Two macroscopic lenses will be provided for the FIR cameras. Both lenses will have motorized focus and zoom features. One lens will be designed to work with the high resolution and ultra high frame rate cameras. This lens will utilize a four tab quick disconnect mount. The lens will accommodate field of views from 14.3 $\mathrm{mm} \times 14.3 \mathrm{~mm}$ up to $100 \mathrm{~mm} \times 100 \mathrm{~mm}$ with at working distances of $120 \mathrm{~mm}$ to infinity. This lens will also have a motorized aperture. The color camera lens will mount to the color camera using a standard Cmount. The lens will accommodate field of views from $2.4 \mathrm{~mm} \times 1.5 \mathrm{~mm}$ up to $67 \mathrm{~mm} \times 43 \mathrm{~mm}$ at working distances from $92 \mathrm{~mm}$ up to $356 \mathrm{~mm}$.

The FIR white light package, Figure 7, will be located on the back of the FIR optics bench. The package will be controlled by the FSAP through the CAN bus. The package consists of two $50 \mathrm{~W}$ metal halide bulbs subassemblies. Each subassembly has a fiber optic quick disconnect for attachment of a fiber bundle. Adjusting an aperture knob located on a subassembly will vary the light intensity delivered through the fiber bundle attached to that subassembly. While the FIR is providing a fiber bundle with a lens attachment, payloads can provide a specialized fiber bundle such as a bundle incorporating a light panel or ring light. Fibers will be routed to the front of the optics bench

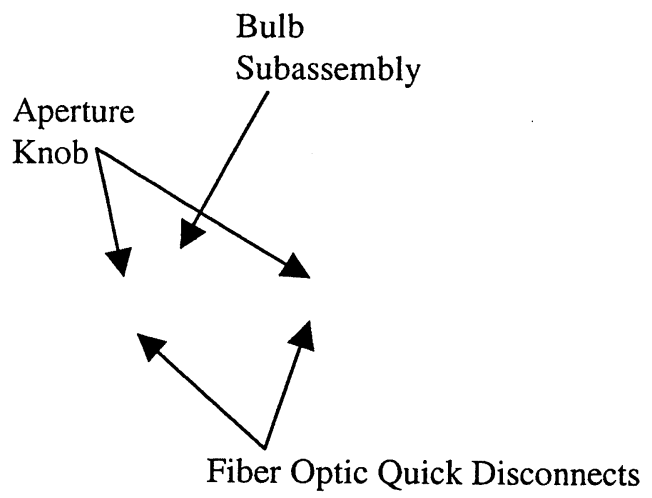

Figure 7 - White Light Package

from the white light package on the back of the bench through the fiber feed through located in the bench.

The FIR $532 \mathrm{~nm} \mathrm{Nd:YAG} \mathrm{laser} \mathrm{package} \mathrm{will} \mathrm{be} \mathrm{located}$ on the back of the FIR optics bench. The package will be controlled by the FSAP through the CAN bus. When using the laser, a fiber optic cable from the laser will be routed from the back of the bench, through the fiber feed through, to payload hardware on the front of the optics bench. A quick disconnect at the end of the fiber optic cable allows for easy hookup to payload hardware. The laser is capable of providing a minimum power of $100 \mathrm{~mW}$ to the payload out of the FIR provided fiber optic cable. The laser output power can be varied from 0 to $100 \%$ of the maximum power. A tap coupler located at the output of laser will measure laser power delivered to the payload.

\section{Typical Diagnostic Techniques Supported By FIR}

As mentioned, there are a variety of experiments to be accomplished in the FIR facility. Potential experiments will encompass a wide range of fluids areas included in the following major fluids theme areas: Complex Fluids; Multiphase Flow and Heat Transfer; Interfacial and Capillary Phenomena; and Dynamics / Stability, and Biofluid Mechanics. Future ISS fluids experiments will fall into one of these theme areas (the first four of which are more fully described reference 3). For example: work in complex fluids covers colloids, foams, granular media, rheology of non-Newtonian fluids, and emulsions and suspensions.

Current schedules show ISS / FIR fluids physics experiments beginning in September 2005. Although it is foreseen that most experiments will be conducted in the FIR, some will be done on other ISS platforms. These include experiments conducted in the EXPRESS rack, the Microgravity Science Glovebox (MSG), and 
in other international facilities such as ESA's Fluid Sciences Laboratory (FSL), and CNES's Dispositif pour l'Etude de la Croissance et des Liquides Critiques (DECLIC). Experimental diagnostics discussed here will focus on various experiments that are planned to be conducted in the FCF/FIR and are summarized below.

Constrained Vapor Bubble Experiment (CVB, P. Wayner - Rensselaer). This experiment will study various heat transfer and flow mechanisms in a constrained vapor bubble heat pipe device.

Physics of Hard Spheres Experiment-2 (PHaSE-2, P. Chaikin - Princeton). This experiment will study nucleation, growth, rheological properties and morphology of hard sphere crystal structures in colloidal suspensions.

Physics of Colloids in Space-2 (PCS-2, D. Weitz Harvard). This experiment will study nucleation, growth, morphology and coarsening, and rheological structural properties of crystal structures in binary alloys, polymer mixtures, and fractal aggregates.

Colloidal Assembly in Binary Particle Suspensions (LфCA, A. Yodh - U. Penn). These experiments will be studying the nucleation and growth of surface crystal structures from low-volume-fraction binary particle colloidal suspensions.

Microgravity Segregation of Energetic Grains ( $\mu \mathrm{gSEG}$, J. Jenkins - Cornell). The experiment will investigate particle segregation in collisional flows of dry granular materials.

Solids interacting with a Gas in a Microgravity Apparatus (SIGMA, M. Louge - Cornell). This experiment will study the interaction between a flowing gas and massive spherical particles with moving boundaries of the cell wall.

Microgravity Obwervations of Bubble Interactions (MOBI, A. Sangani - Syracuse). This experiment will attempt to validate the theory for bubble interactions in a bubbly suspension in a constant shear environment.

Nucleate Boiling in Microgravity (NBX, V. Dhir UCLA). This experiment will attempt to develop an understanding of heat transfer, vapor removal processes during nucleate boiling conditions.

Microscale Hydrodynamics Near Moving Contact Lines ( $\mu$ SCALE, $S$. Garoff - Carnegie Mellon). This experiment will study flow fields and interface shapes very close to a moving dynamic contact line.
Many of these experiments will utilize mini-facilities. These mini-facilities will be resident on the FIR and will be able to accommodate a series of experiments prior to returning to the ground. The first four experiments will be done in the Light Microscopy Module $^{4}$. The fifth and sixth experiment will be done in the Granular Fluids Module ${ }^{5}$. These experiments require a variety of diagnostics techniques ranging from simple imaging to confocal microscopy. A summary of these techniques follows:

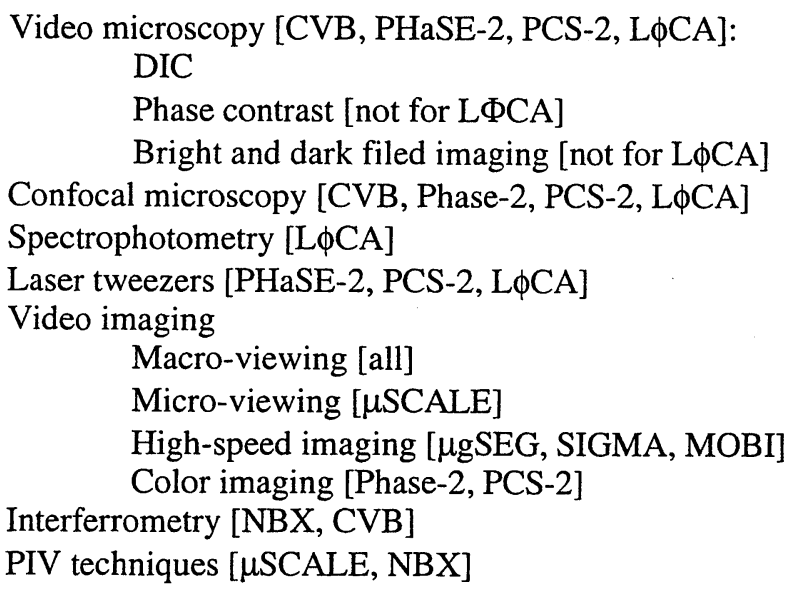

In general, the diagnostic packages provided by the FIR will have to be augmented by PI supplied hardware in order to meet unique science requirements. Examples of such latter type of hardware would include beam shaping optics, beam splitters, and specialized light sources.

The diagnostics required for the FIR experiments mentioned above are described below. Examples of data output for selected diagnostics are also given.

Video microscopy

This basic capability will be used in the LMM by the PHaSE-2, PCS and L $\phi C A$ experiments to observe colloidal structures and dynamics through the various modes and magnifications levels. The nucleation and growth morphology of the evolving crystals will be studied. There are two modes of illumination and visualization: the epi-illumination and transillumination modes. The former uses light that is reflected off the sample; whereas the latter mode transmits light through the sample from below. There are various types of video microscopy that all use the trans-illumination technique: bright and dark field, phase contrast, and DIC. Facility supplied light sources and cameras will be used by LMM to support video microscopy. In addition, image data can be subjected to computer image analysis in the IPSU. 


\section{Confocal microscopy}

Confocal microscopy is required for PHaSE-2, PCS-2 and L $\phi$ CA. Confocal microscopy will be implemented by LMM using a $532 \mathrm{~nm}$ frequency-doubled Nd:YAG laser, a confocal scanner, and a 12-bit digital CCD camera. The laser will be the FIR laser. This method uses a spinning array of apertures and lenses to individually map regions of the sample onto the CCD array. The rotational speed of the scanner will allow 30 frames per second of confocal images to the CCD camera. The crystal three-dimensional structure is reconstructed by assembling the slices with an image analysis program, from which colloidal growth, structure, and dynamics can be measured. These images will be stored in the facility IPSU for later downlinking or archival on the IOP hard dives. Figure 8 is an example of a confocal image of colloidal particles, 2.3 micron diameter, with a fluorescent dye.

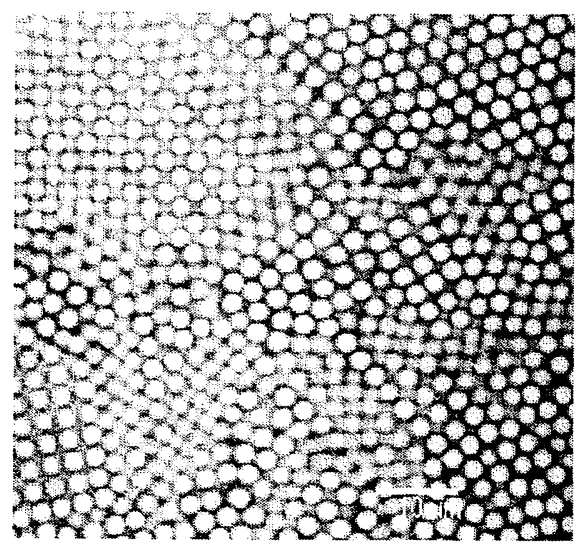

Figure 8 - Confocal Microscope Image

\section{Interferrometry.}

Interferrometry is required by CVB. Epi-illumination is used for this diagnostic. LMM will use the FIR provided White Light Package that provides the wavelengths required by this diagnostic technique. The wavelengths reflecting off various surfaces and interfaces (e.g., glass liquid interface and liquid / vapor interface) provides the interference fringes required for analysis. Figure 9 shows interferrometric fringe patterns from a critical point experiment. There are other types of interferrometry that can be supported by FIR light sources and cameras. Specific interferrometric optics would have to be provided by the payload. Examples include Mach-Zehnder, Michelson, and Twyman-Green interferrometry. Interferrometry can be sensitive to vibration and air flow within the rack. The ARIS rack isolation system, in the FIR, will minimize vibrations. Payloads can also employ encapsulations or baffling approaches that can address air flow concerns.

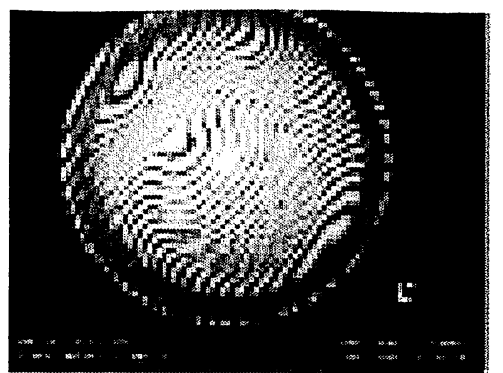

Figure 9 - Interferrometric Fringe Patterns

\section{Spectrophotometry}

Spectrophotometry is required by L $\phi C A$ and PCS-2. This diagnostic is used to assess the photonic band gap structures of a crystal. Light from broadband sources is filtered, chopped and polarized. The incident light is then sent to a splitter, and the light intensity is measured through the sample (photo diode). A ratio of the relative intensities of the incident light and the light that passes through the sample is made. This ratio is studied as a function of the angle of incidence as well and wavelength. The band structure of the photonic crystals is thus determined. In implementing spectrophotometry LMM will be providing the photo diodes and utilizing the FIR $532 \mathrm{~nm}$ Nd:YAG laser.

\section{Laser tweezers}

Laser tweezers are required by PHASE-2, PCS-2 and L $\phi C A$. A laser tweezer is a device for trapping of a colloidal particle using radiation pressure by focusing a laser beam through a high-numerical aperture lens. This diagnostic will be employed to support rheological type measurements; such as displacing particles from their equilibrium positions. Laser tweezers also will be used to measure the viscosity of the fluid (e.g., a particle is trapped and video images taken as it is translated in an oscillatory fashion through the field of view). The capability will be implemented by LMM using a custom-built system based upon a $1064 \mathrm{~nm}$ Nd:YAG laser, beam focusing optics, and two acoustooptic deflectors to steer the trap within the field of view of the microscope. Figure 10 shows a 10 micron polystyrene sphere in a 0.956 micron PMMA-decalin suspension. Optical tweezers were used to hold the

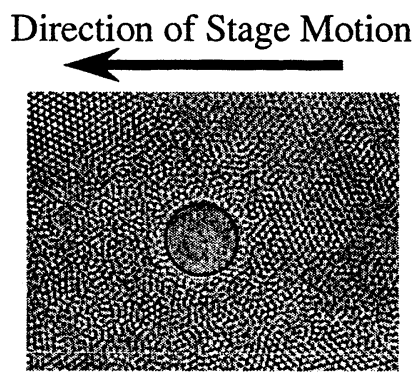

Figure 10 - 10 Micron Polystyrene Sphere Being Dragged Using Laser Tweezers

large sphere fixed in location while the sample stage was dragged as indicated by arrow.

Video imaging / Macro-viewing 
Many, if not all, experiments will be using the basic video imaging capabilities of the FIR. The cameras and attached lenses will be used along with the appropriate light sources to image various phenomena. In the macro lens mode, fields of view on the order of 2 through $10 \mathrm{~mm}$ are accommodated.

\section{Video imaging / Micro-viewing}

Many experiments will also be using the basic video capabilities of the FIR in the micro imaging mode. Although, perhaps providing fields of view that are commensurate with the LMM microscope, the FIR supplied micro-imaging capability can accommodate much larger sample sizes. The FIR supplied cameras and attached lenses are used along with the appropriate light sources to image the phenomena. In the micro lens mode fields of view on the order of 200 microns through $2 \mathrm{~mm}$ are accommodated.

\section{Video imaging / High-speed imaging}

There are several experiments, such as $\mu \mathrm{gSEG}$ and SIGMA, that require frame rates significantly higher than the standard 30 frames per second. The FIR cameras will be available to provide up to on the order of 1000 frames per second for up to 4 seconds (and for

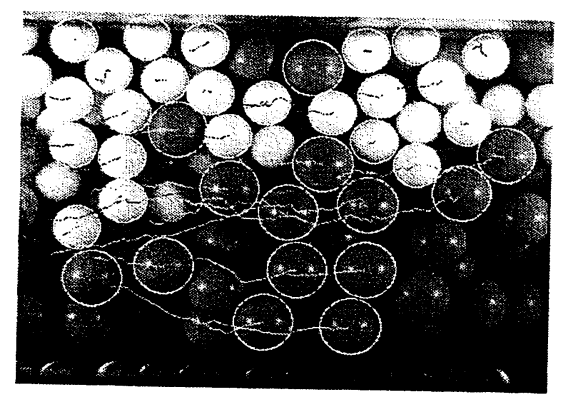

Figure 11 - Post Processed High Frame Rate Images of a Binary Mixture of Particles

longer duration at decreasing pixel density) for these studies. Shown in Figure 11 are images of a binary mixture of particles for the $\mu \mathrm{gSEG}$ experiment, moving at relatively high speeds, taken using a high frame rate camera. Through post processing the particle trajectories can be determined as shown in Figure 11.

One challenging aspect for this diagnostic is that the high image data flows cannot be accommodated by an IPSU. They must be stored in the camera memory; then, downloaded to the IPSU post test. The camera memory will limit the length of time the test can run; and the download rates to the IPSU limit the turnaround times. These limitations will affect the test matrices that can be accommodated.

\section{PIV techniques}

PIV will be used by $\mu$ SCALE. These diagnostics are typically used to measure velocity fields. The fluids are seeded with small particles that reflect light from a laser light sheet. A PIV image is shown in Figure 12.

Light sheet optics will have to be provided by the payload; whereas facility cameras and lasers can

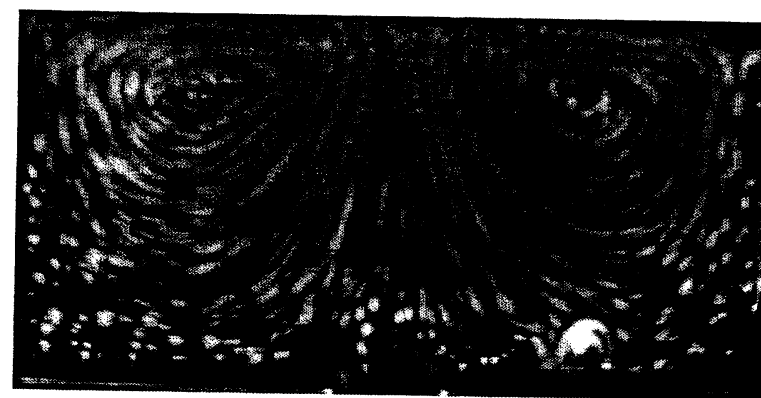

Figure 12 - A PIV image showing the sub-surface flow from the Surface Tension Driven Convection Experiment (STDCE).

typically meet the illumination requirements.

\section{On-Orbit Operations}

After the initial installation and checkout of the FIR, on-orbit operations will consist mainly of maintenance and payload operations. Maintenance operations include things. such as filter cleaning and/or replacement, replacement of failed or degraded hardware and recalibration of hardware.

In order to minimize crew time FIR packages or package subassemblies are designed as orbital replacement units (ORU's). In some cases an ORU is a package such as the FSAP, in other cases an ORU may be a sub package component, such as the white light package bulb assembly. In case of failure or performance degradation, facility hardware will be replaced at the ORU level. On-orbit sparing of individual packages or ORU's will be based on the results of reliability analysis for each package.

Periodic recalibration may be necessary for certain FIR diagnostics and optics. The white light package is one example of a package that will require periodic calibration. The intensity of the white light will change over time requiring the intensity at the various aperture settings to be periodically checked.

On-orbit payload operations begin by initially configuring the FIR for a specific payload. The optics bench can be translated out and any FIR diagnostic hardware not required for a payload can be removed and stowed. Next the payload hardware can be removed from stowage and installed on the front of the optics bench. Rotating the bench forward will allow inner-connecting cables, such as fiber optic cables, from the back to the front of the bench to be run. Once 
the payload hardware is installed and all cables and hoses have been run the bench can be slid back into the rack and the rack doors can be closed. Once the doors are closed the FIR can be powered up.

Due to limited crew time, most of the powered up payload operations will be conducted from the Telescience Support Center (TSC) at the Glenn Research Center. A ground team made up of both the FIR and payload teams will operate the FIR and payload hardware from the TSC. These teams will be able to monitor the health and status of the FIR and payload hardware, issue commands and review data in near realtime and post test. Once testing is completed the payload hardware will be removed and stowed until it can be returned to earth.

\section{Summary}

The FIR will provide common laboratory diagnostic hardware, in a flexible environment, in order to accommodate a variety of fluid physics experiments. The optics bench will allow for precision mounting of diagnostic hardware. In addition to providing a suite of diagnostic tools for use by the payloads, the FIR's flexible interfaces will allow payloads to utilize experiment unique diagnostic hardware.

The FIR's avionics provides a customizable control and data acquisition system. The FIR avionics can be augmented by payload unique data acquisition and control hardware. For experiment control, the system will allow payload specific software to be uploaded providing the unique control algorithms required to operate the FIR and payload hardware.

The design of the FIR minimizes the use of the crew. The use of ORU's in the design of the FIR will allow for easy maintenance and upgrades of the FIR hardware. The translating optics bench and quick disconnect interfaces allow for easy installation and removal of FIR and payload hardware. Once the FIR is configured for a particular payload and the payload hardware is installed the FIR will be operated from the ground requiring the crew only for sample change out and troubleshooting.

\section{Acknowledgements}

The FIR is being developed within the Microgravity Science Division of the NASA Glenn Research Center. The authors of this paper would like to acknowledge the hard work and dedication of Northrop Grumman development team. It is through their dedication and innovativeness that the FIR will be a unique facility for carrying out fluids physics experiments on board the International Space Station.

\section{References}

1. Zurawski, R.L., "The ISS Fluids and Combustion Facility: Microgravity Combustion Science and Fluid Physics Research Capability", AIAA-2001-4925, Conference \& Exhibit on International Space Station Utilization-2001, October 2001.

2. O'Malley, T. and Weiland, K. "The Fluids and Combustion Facility Combustion Integrated Rack: Microgravity Combustion Science on Board the International Space Station", AIAA-2001-4927, Conference \& Exhibit on International Space Station Utilization-2001, October 2001.

3. Singh, B. and Alexander, J.I.D., "Microgravity Fluid Physics and Transport Phenomena Experiments Planned Aboard the International Space Station", STAIF-2000, Alburquerque, NM, February 2000.

4. Motil, S. and Snead, J., Hovenac, E. "Light Microscopy Module Multi-User Hardware Description", AIAA-2001-4956, Conference \& Exhibit on International Space Station Utilization-2001, October 2001.

5. Caruso, J., "Granular Flow Module Multi-User Hardware Description”, AIAA-2001-4990, Conference \& Exhibit on International Space Station Utilization2001, October 2001. 